EPOS, XIII (1997), págs. 13-29

\title{
SINTAXIS Y ARGUMENTACIÓN EN EL CONTRA CTESIFONTE DE ESQUINES
}

\author{
Marfa José Martín Velasco \\ Universidad de Santiago de Compostela
}

\section{RESUMEN}

Este trabajo pretende ofrecer un análisis de los períodos hipotéticos que encontramos en el Contra Ctesifonte de Esquines, con el fin de estudiar si este orador utiliza dichos períodos habitualmente como formas de entimemas. Se pretende con ello profundizar en los mecanismos de persuasión utilizados por los oradores y su relación con la normativa que presenta Aristóteles en la Retórica. En los períodos hipotéticos podemos descubrir esquemas silogísticos que siguen un esquema constante: prótasis como premisa menor y apódosis como conclusión en las condicionales reales y prótasis como conclusión y apódosis como premisa menor en las irreales. La premisa mayor de carácter probabilístico está generalmente omitida.

Intentamos con este trabajo profundizar en el estudio de la argumentación retórica a partir de los esquemas sintácticos frecuentemente utilizados por los oradores como formas de entimemas. En esta ocasión analizamos los períodos hipotéticos encontrados en el Contra Ctesifonte de Esquines '.

1 Remitimos, para una comprensión más clara de esta investigación, a los trabajos de CoRTÉs GabaudÁN, Francisco (1994) «La interpretación del texto: Lysias 24. (Lectura aristotélica de la 
Vamos a ofrecer primero unas ideas generales sobre la concepción aristotélica de la retórica y en concreto del entimema. Aristóteles afirma que la

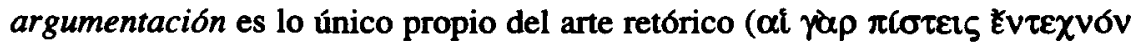

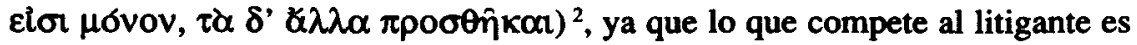
mostrar al juez «que eso es así o no es, probar si algo existió o no existió» ${ }^{3}$. Todo lo demás, como «el mover a sospecha, a compasión, a ira y a otras pasiones semejantes del alma" ${ }^{4}$, «el prescribir reglas sobre qué debe contener el exordio o la narración y cada una de las otras partes» ${ }^{5}$, como han hecho otros tratadistas, son cosas ajenas al asunto, porque van dirigidas a disponer al juez en un sentido determinado, lo cual equivale a «torcer la propia regla de la que uno se ha de servir» ${ }^{6}$. Más adelante ${ }^{7}$ establece Aristóteles una distinción entre $\pi\left\{\sigma \tau \varepsilon \varsigma^{8}\right.$ \% $\tau \varepsilon \chi v o r$, «las que no se obtienen por nosotros, sino que existían de antemano, como los testigos, las confesiones bajo suplicio, los documentos y

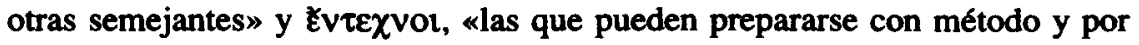
nosotros mismos». Las primeras pueden también transformarse en $\varepsilon^{\prime} v \varepsilon £ \chi v 0{ }^{9}$ y ser susceptibles de un tratamiento ret6rico. Las pruebas $\varepsilon v \tau \varepsilon \chi v o l$ son a su

argumentación)», Actas de las IX Jornadas de Filología Griega, Cáceres y (1994) «Formas y funciones del entimema en la oratoria ática», CFC, pp. 226-225, en cuyo marco de investigación se encuadra nuestro estudio. Allr se recogen con detalle las distintas interpretaciones del texto aristotélico y las referencias bibliográficas que, en líneas generales, hemos seguido.

${ }_{2}$ Arist. $R h .1354$ a 14. Traducimos aquí el término $\pi l \sigma \tau \varepsilon l \varsigma$ por argumentación, entendida como la finalidad del proceso argumentativo, es decir, como la convicción que se produce en el oyente. Más adelante veremos que este mismo término adquiere el sentido de recursos técnicos con los que se argumenta y de fuentes de enunciados. Cf. para su comprensión los artículos ya citados de Cortes, en los que sigue en este punto la interpretacion de GrImaLd, William (1972), Studies in the Philosophy of Aristotle's Rhetoric, Wiesbaden y (1980) Aristotle, Rhetoric I, A Commentary, New York. Cf. igualmente RACIONERo, Quintín (1990), Aristoteles. Retórica, ed. Madrid, Gredos; cuya traducción hemos seguido en líneas generales.

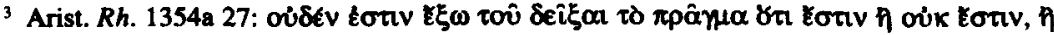

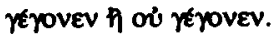

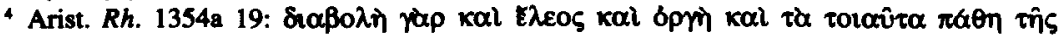

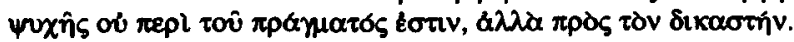

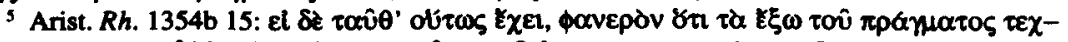

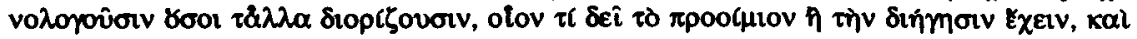

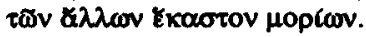

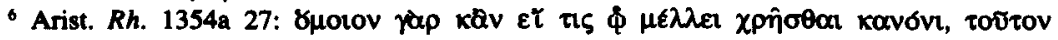

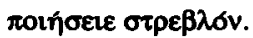

' Arist. Rh. $1355 \mathrm{~b} 35$.

- Aqui el término $\pi i \sigma \pi \zeta$ que traducimos por pruebas adquiere el sentido de fuentes de enunciados de donde proceden las proposiciones persuasivas ( $\pi$ lotelৎ), con las que se consigue la persuasion ( $\pi$ lotis).

${ }^{9}$ Arist. Rh. 1375a 25. 
vez de tres especies «unas residen en el talante del que habla ( $\varepsilon v \tau \hat{\varphi} \not \xi \theta \varepsilon$

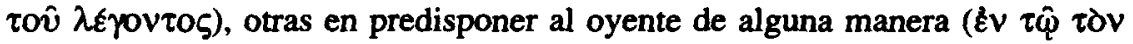

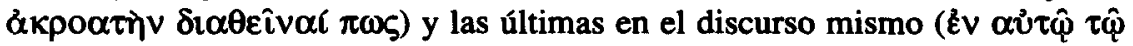
$\lambda \sigma \gamma()_{)}{ }^{10}$. En otro pasaje ${ }^{11}$ afirma que «la prueba es una especie de demostra-

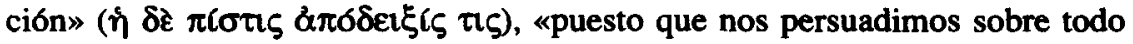

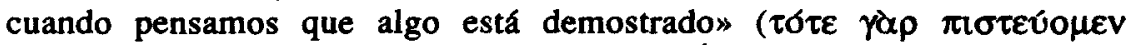

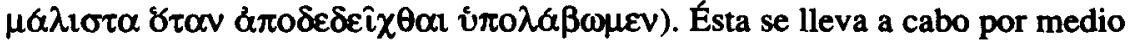
de entimemas y de ejemplos, que son los equivalentes en la dialéctica al silo-

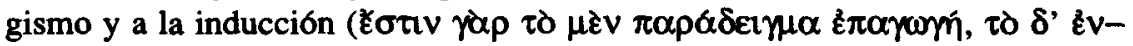

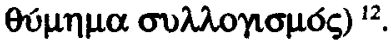

El entimema es, por tanto, un silogismo retórico semejante al dialéctico, con el que se obtiene, «dadas ciertas premisas, algo diferente de ellas» ${ }^{13}$. Se diferencia del dialéctico en que en el entimema las premisas con las que argumenta son probables ${ }^{14} \mathrm{y}$ en el silogismo necesarias. Es decir, se razona de una forma general, partiendo de pautas habituales en el comportamiento humano y llegando, en consecuencia, a una conclusión también sólo probable. Por tanto, la principal tarea del orador será la de buscar esas premisas basadas en el elkó̧, en las que poder apoyar la argumentación ${ }^{15}$. Los entimemas, por otra parte, se presentan de forma abreviada, sin alguno de los elementos del silogismo. Lo más frecuente es que esté omitida la premisa mayor, que el orador da por supuesta por considerarla opinión común de los jueces, pero también pueden ser la premisa menor o incluso la conclusión las que falten. Por eso no son fáciles de reconocer en el discurso y a veces hay que hacer una especie de reconstrucción del silogismo añadiendo elementos tomados del contexto.

Lo que nos proponemos estudiar ahora es si determinados modelos sintácticos, en nuestro caso los periodos hipotéticos, son formas en las que habitualmente se presentan entimemas. Nos hemos centrado en esta construcción porque es una de las que utiliza Aristóteles con frecuencia ${ }^{16}$ y podemos suponer que los oradores la usasen igualmente para la formulación de entimemas. El análisis de las condicionales se centrará tanto en la forma, es decir, en la reconstrucción del proceso argumentativo, como de la función. El proceso argumentativo, como

\footnotetext{
10 Arist. $R h .1356$ a 13.

$"$ Arist. Rh. 1355a 4, $\pi$ loteı $\zeta$ aquí como recursos técnicos con los que se argumenta.

12 Arist. Rh. $1356 b 1$.

13 Arist. $R h .1356 \mathrm{~b} 18$.

14 Arist. $R h .1357$ a 23.

is Arist. $R h .1395 b 25$.

16 Arist. $R h$ II. Cap. 23 y 24.
} 
explica Hood ${ }^{17}$, consiste en una aserción particular (conclusión del silogismo) que, en el caso de un discurso judicial, hay que demostrar a los jueces apoyándose en algo generalmente admitido (premisa mayor del silogismo), buscando un punto de conexión entre ambos (premisa menor). Pues bien, en las condicionales ${ }^{18}$ la prótasis corresponde la mayor parte de las veces a la premisa menor del silogismo en las reales y la apódosis a la conclusión, mientras que en las irreales la prótasis es la conclusión y la apódosis la premisa menor. La premisa mayor falta en muchos casos y hay que reconstruirla con elementos del contexto. Es frecuente encontrar también la inserción de ejemplos que participan de la estructura del entimema introduciendo un principio general que confirma el caso particular, es decir, como enlace de la premisa menor y la premisa mayor ${ }^{19}$. Haremos también referencia a su relación con los tópicos en cuanto que nos sugieren la forma de integración en el proceso formal de la argumentación ${ }^{20}$. En el estudio de la función ${ }^{21}$ trataremos de ver cual es la declaración general de naturaleza probable que sirve de punto de partida, con la que el orador supone que el auditorio está de acuerdo y la esfera a la que hace referencia.

Dentro de las condicionales no vamos a tratar de las de futuro (eventuales, reales y potenciales) o proyectadas hacia el futuro (con imperativo o con expresiones del tipo $\beta o u ́ \lambda o \mu \alpha$, , $\delta \varepsilon \hat{\imath}$ etc...), ya que en este discurso hacen siempre referencia a las consecuencias de la votación, a posibles manifestaciones del adversario o al desarrollo del discurso, pero no a la argumentación y que se encuentran situadas en lugares de transición, en el exordio y en el epílogo. Esto confirma lo que dice Aristóteles de que el futuro es el tiempo adecuado a los deliberativos y el pasado el de los judiciales, ya que éstos argumentan sobre hechos ya ocurridos ${ }^{22}$. Respecto a las condicionales generales hay que decir que, tanto por su carácter general como por la estrecha conexión semántica de sus dos miembros ${ }^{23}$, difícilmente se ajustan al proceso argumentativo de los entimemas. Vamos a tratar de ellas solo en un uso frecuente en este discurso que es el de la paráfrasis de leyes y que usaremos como ejemplo de la transformación

17 M.D. Hood, (1984), Aristotle's Enthymeme: its Theory and Application to discourse, Un. de Oregón, (UMI 1990).

18 Remitimos de nuevo a los artículos de Cortés Gabaudán, citados en la nota 1, en la que recoge algunos ejemplos de entimemas presentados en forma de condicionales en oradores áticos.

19 Cf. HоOHD p. 47.

20 Cf.Grimaldi (1972), p. 115.

21 Seguimos en esto a Cortés Gabaudán (1994), CFC.

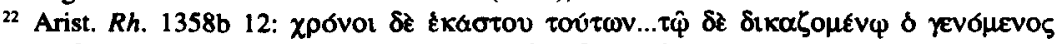

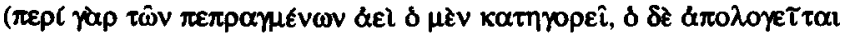

${ }^{23}$ Cf. BAKKeR, E. J. (1986), «Restrictive conditionals», In the footsteps of Raphael Kühner, Amsterdam, pp. 5-26. 


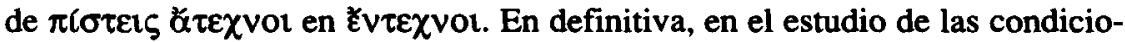
nales como posibles modos de presentarse un entimema nos limitaremos a analizar las reales e irreales de pasado y a algunas reales de presente. No es esto sorprendente si consideramos que la relación que se establece entre la prótasis y la apódosis en los periodos hipotéticos de indicativo es la menos subjetiva y, por tanto, la más adecuada para crear relaciones lógicas entre sus dos miembros ${ }^{24}$.

Analizamos ahora el discurso. Estamos ante una ypaфń $\pi \alpha \rho \alpha v \sigma \mu \omega v$, es decir, una acusación por ilegalidad contra el decreto que Ctesifonte, político amigo de Demóstenes, había conseguido ya hacer llegar a la asamblea y en el que proponía que a Demostenes se le otorgara una corona de oro por los grandes servicios prestados a la ciudad y por haber contribuido con su dinero particular a la reconstrucción de las murallas, cuando fue nombrado inspector de fortificaciones. Esquines desarrolla las acusaciones formuladas contra Ctesifonte, aunque, en realidad, van dirigidas a Demóstenes.

En el exordio ( $\$ 1-8)$, donde Esquines hace una defensa de existencia de las $\gamma \rho \alpha \phi \eta ́ n ~ \pi \alpha \rho \alpha v \delta \mu \omega v$ no se encuentra ninguna condicional que encubra entimema. Sí, sin embargo, una condicional general de presente (\$3), una real de futuro (\$5) y dos eventuales $(\$ 8)$. Estas tres últimas haciendo referencia al desarrollo del discurso.

Prosigue el discurso con la demostración de la ilegalidad del decreto de Ctesifonte (\$\$9-48) argumentando que Demóstenes aún no ha hecho rendicion de cuentas por la magistratura que ha ejercido (\$\$9-31) y que el decreto propone que la proclamación se haga en el teatro y no en la Pnix (\$\$32-48). Esquines utiliza para la paráfrasis de leyes dos condicionales generales de presente. Aris-

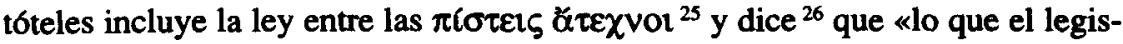
lador ha dejado sin explicitar, eso conviene que lo determine el mismo juez» y que «es sumamente importante que las leyes estén bien establecidas, hasta donde sea posible, por si mismas, y que dejen cuanto menos mejor al arbitrio de los que juzgan». Es decir, en principio, donde acaba la ley, empieza la argumenta-

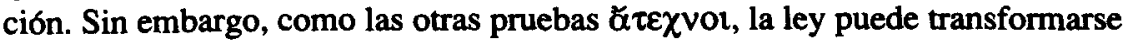
en $\varepsilon v \tau \varepsilon \chi v{ }^{27}{ }^{27}$ y eso es lo que ocurre en esta parte del discurso en la que lo que importa es mostrar la disconformidad del decreto con la ley:

${ }^{24}$ Cf. BASSET, Louis (1989), La syntaxe de l'imaginaire, Paris, y WAKKER, G. (1994), Conditions and Conditionals, an investigation of Ancient Greek. Amsterdam.

25 Arist. Rh. 1375a 22.

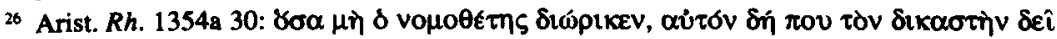

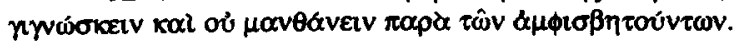

27 Arist. $R h$. 1375 a25 y $1375 b$ 


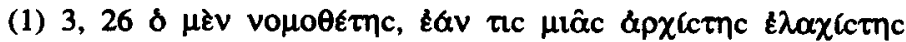

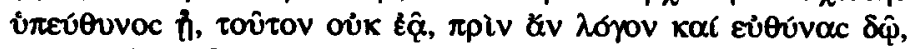

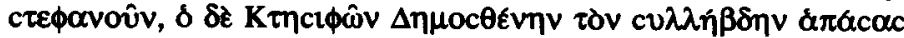

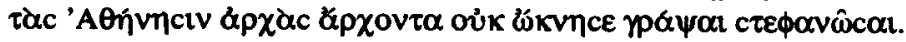

El período hipotético proporciona los dos términos de la premisa mayor:

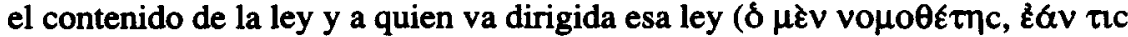

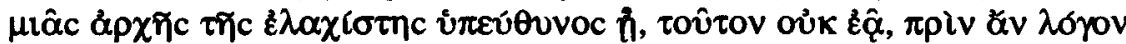

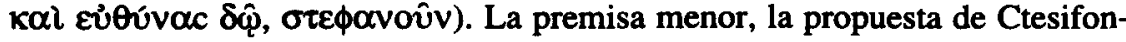
te, está también explícita en una oración coordinada con la condicional ( $\delta \delta \varepsilon$

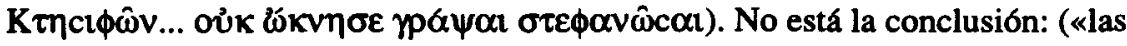
leyes que regulan la rendición de cuentas han sido violadas por Ctesifonte»), pero está suficientemente clara, ya que es aquello de lo que está tratando des-

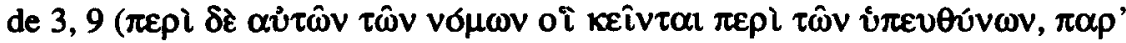

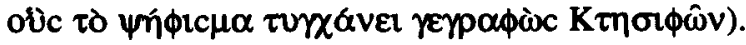

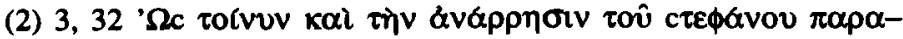

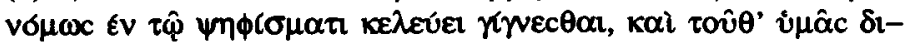

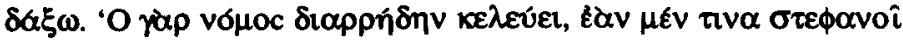

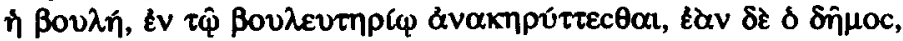

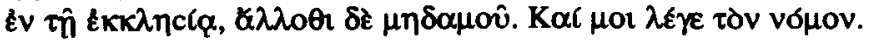

En este caso la aserción sí que está expresa, pero fuera del periodo hipotético: «la proclamación de la corona propuesta en este decreto es ilegal» ( $\tau \grave{\eta} v$

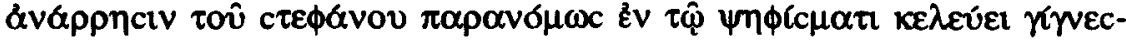

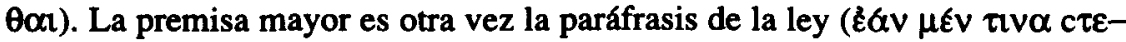

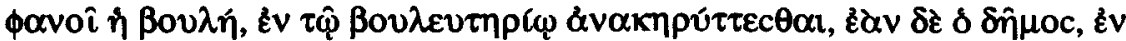

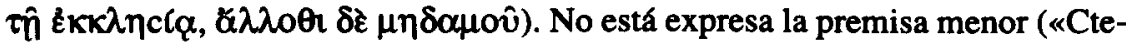
sifonte propone hacer la proclamación de una corona que otorga el pueblo en el teatro»), que explicará después.

Las condicionales generales de presente, así como las eventuales, se caracterizan semánticamente porque completan el enunciado de la oración principal que, sin la condicional, quedaría indefinido ${ }^{28}$. Esto es lo que, al situarlas en un contexto presente, les otorga el valor de iteración. El carácter general de este tipo de condicionales y el hecho de que el sentido de la apodosis quede incompleto sin la prótasis nos impide dividir sus dos miembros y reconstruir una premisa particular, con lo que difícilmente se ajustan al esquema fijado

${ }^{28}$ Cf. al respecto BAKKER (1986) y WAKKER, G. (1986), «Potential and Contrary-To-Fact Conditional in Classical Greek», Glotta 64, pp. 222-246. 
al principio. Teniendo además en cuenta lo que explica en otro pasaje, que «el juicio del legislador no versa sobre lo particular, sino que trata sobre lo futuro y universal, mientras que el miembro de una asamblea y el juez tienen que juzgar inmediatamente sobre casos presentes y determinados ${ }^{29}$, no es extraño que sean las adecuadas para servir de premisa mayor para una argumentación apoyada en la ley.

Los tres entimemas siguientes, basados en condicionales, que se encuen-

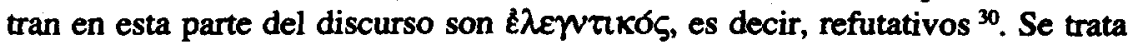
de hacer ver la contradicción de los términos: «este sostiene que..., pero su afirmación se contradice con el hecho de que...» y están introducidos por expresio-

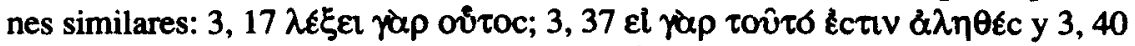

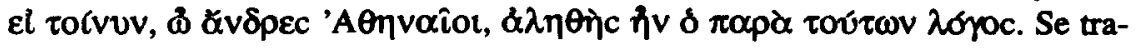
ta de dos condicionales reales de presente (3) y (4) y una irreal de pasado (5).

En 3, 17 se anticipa Esquines a la posible defensa de Demóstenes (cf. D $18,110-119)$ de que no tiene que dar cuenta de ese dinero porque, en realidad, no era dinero público sino una aportación suya:

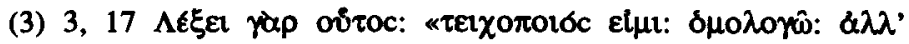

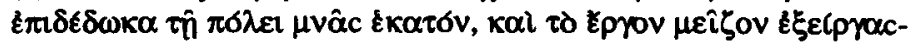

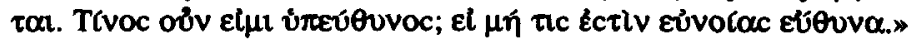

(«No hay que dar cuenta de los regalos»), «yo he hecho un regalo» (El $\mu \hat{\eta}$

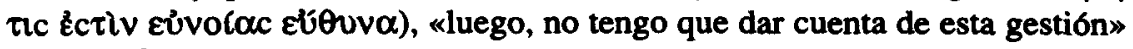

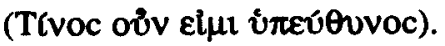

Los otros dos se refieren al lugar de la coronación. Ctesifonte aducirá que existen dos leyes: la que ahora se cita y otra que permite hacer la proclamación en el teatro en las representaciones de tragedias, si el pueblo lo decreta. Esquines argumenta que la constitución tiene mecanismos capaces de detectar si existen dos leyes sobre el mismo tema que se anulan mutuamente y de abolir una de ellas si se hubiera dado el caso:

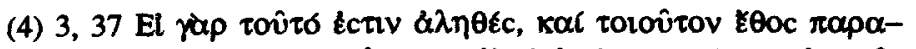

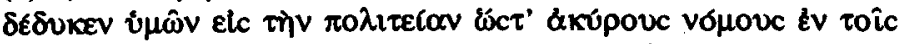

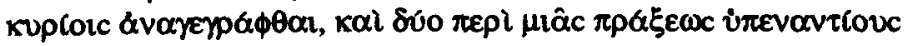

29 Arist. Rh. 1354b1-15.

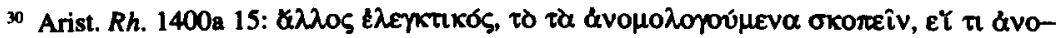

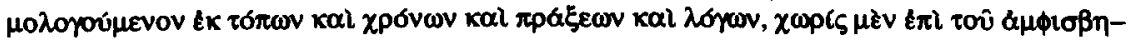

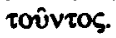




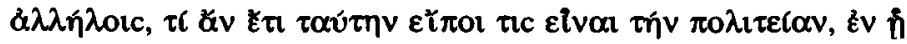

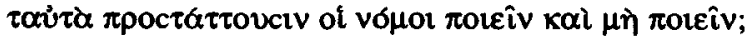

(«Las constituciones no admiten leyes contradictorias»), «éste dice que

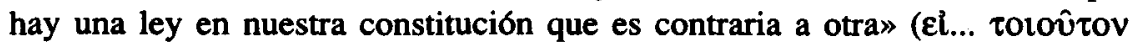

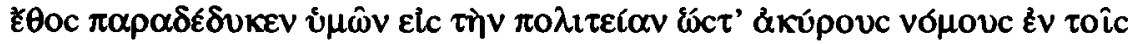

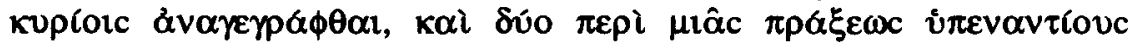

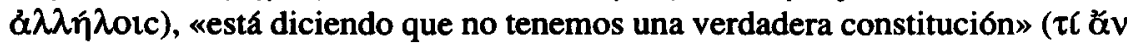

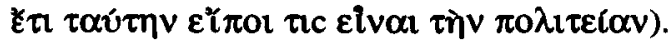

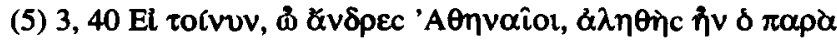

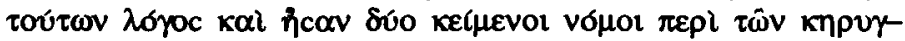

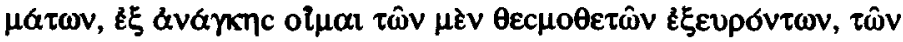

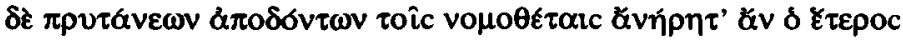

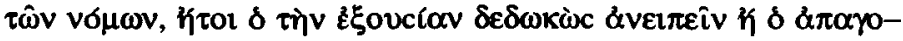
pev́wv.

(«Las ciudades evitan las leyes contradictorias suprimiendo una de

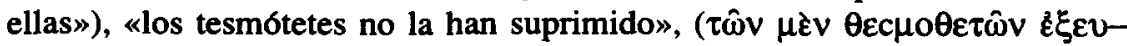

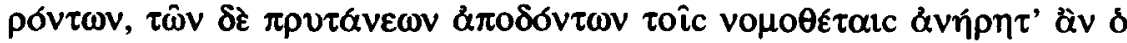

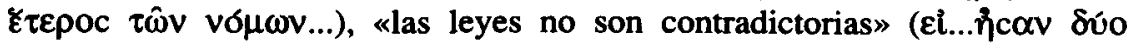

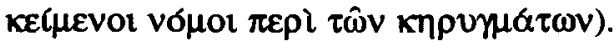

Entramos ahora en el momento en que Esquines repasa las sucesivas etapas de la actuación pública de Demóstenes (\$\$49-167) para demostrar que no merece el honor para el que está propuesto, ya que no ha obrado siempre en favor de la ciudad, sino que, en muchos momentos, la ha puesto en trance de peligro haciéndole correr riesgos innecesarios. Los tres primeros entimemas (6), (7) y (8) reconstruidos sobre condicionales que encontramos en este fragmento del discurso, lo están sobre irreales de pasado y argumentan afirmando en el plano de lo real lo que es una causa solo posible. Hacen referencia al tópico

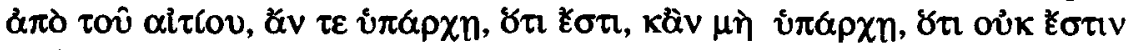
(Arist. Rh. 1399b 20).

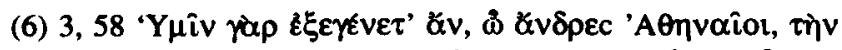

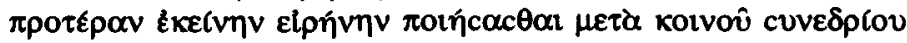

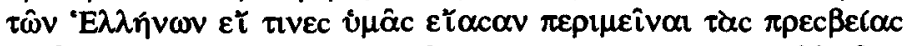

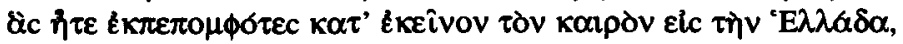

(«Firmar la paz, no solos, sino con la conformidad de los otros estados es lo que produce una situación de hegemonía»), «Demóstenes impidio que fir-

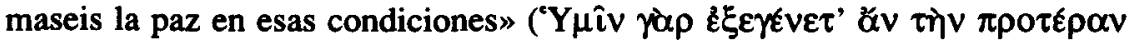




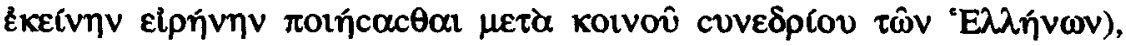

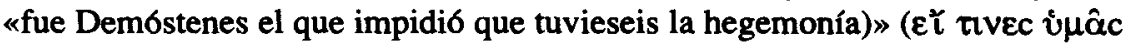
$\varepsilon^{2}(\alpha c \alpha v)$

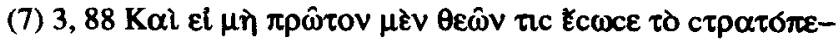

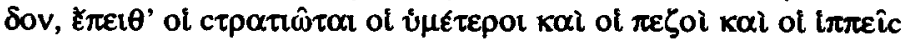

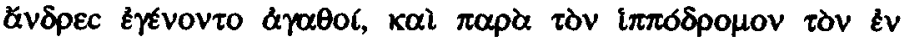

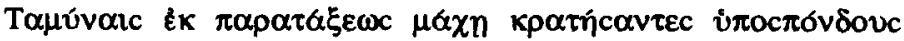

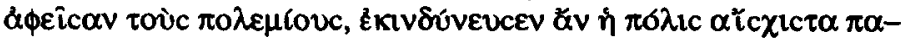
$\theta \varepsilon i ̂ v$.

( $\ll$ Las situaciones de extremo peligro exigen un comportamiento extraor-

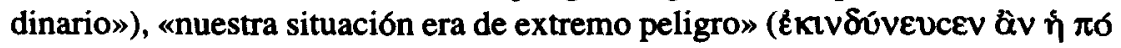

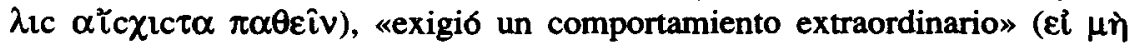

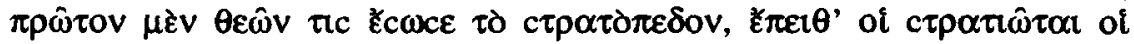

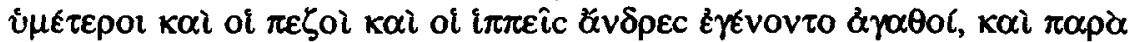

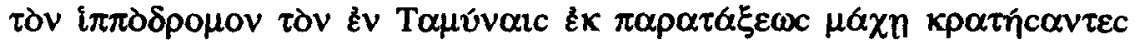

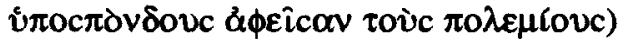

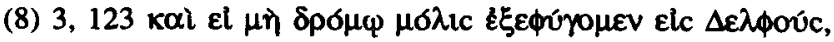
ยส

(«El recurso a la huida es propio de situaciones de extremo peligro»), «nos

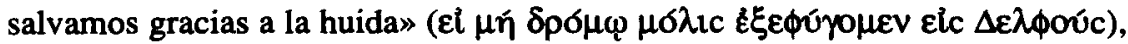

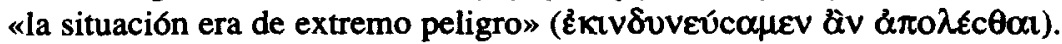

Siguen tres condicionales reales de pasado (9), (10), (11). En (9) y (10) se nos ofrece un caso de ejemplo integrado en el entimema, como punto de unión de la premisa mayor y la menor ${ }^{31}$. En (9) el modo es el de $R h 1397 \mathrm{~b} 13$.

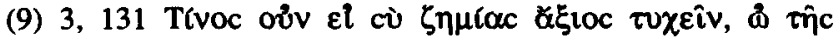

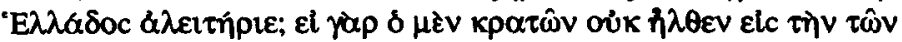

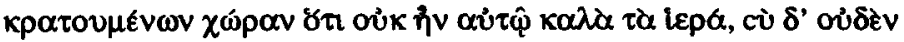

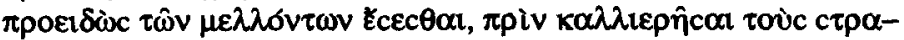

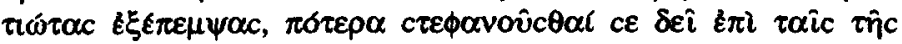

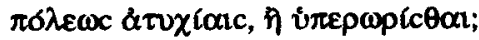

(«Son enemigos de la ciudad los que permiten que corra riesgos innecesarios»), «el poderoso no fue al lugar enemigo porque no tenía las víctimas a

${ }^{31}$ Cf. HoOD, p. 47. 


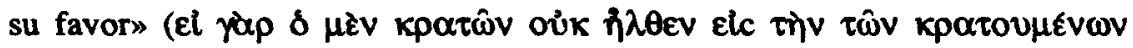

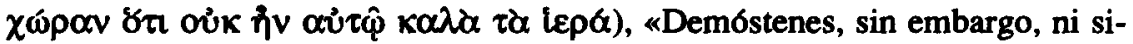

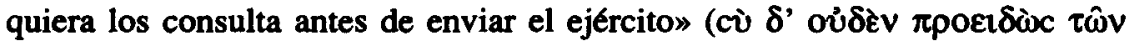

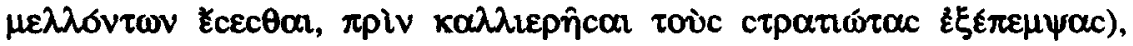
«Demóstenes es enemigo de la ciudad y merece la expulsión» ( $\pi \delta \tau \varepsilon \rho \alpha$

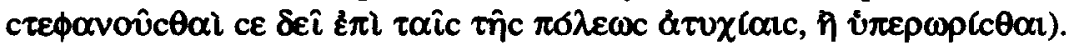

En (10) la inducción es similar al que se recoge en Arist. $R h .1_{1398 b 1}{ }^{32}$.

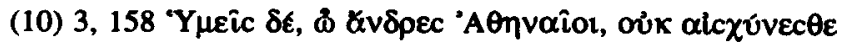

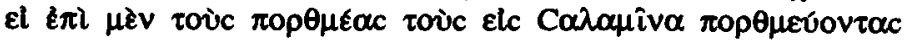

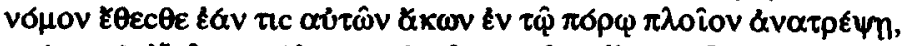

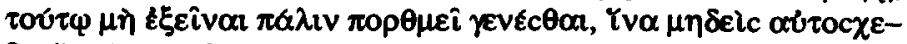

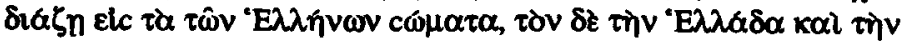

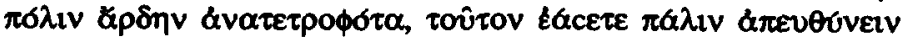
$\tau \grave{\alpha}$ kotva;

( No debe seguir dirigiendo quien ha hecho fracasar aquello que dirigía»), «A los barqueros que hacen la travesía a Salamina la ley les prohibe ejercer de nuevo su profesión si una vez, involuntariamente, vuelcan la barca» (Tờc

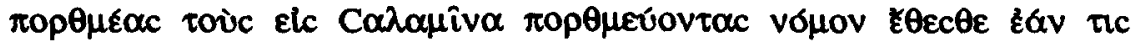

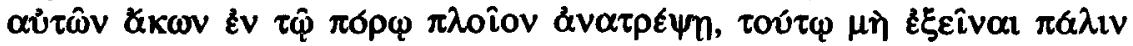

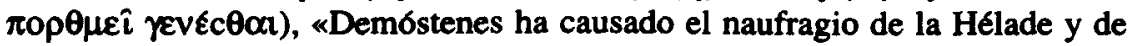

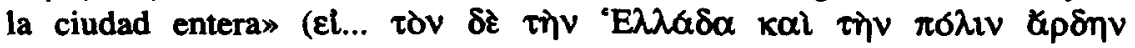
\&v $\alpha \tau \varepsilon \tau \rho \phi \sigma \tau \alpha)$, « Demóstenes no debe seguir al frente de los asuntos públicos» (

El siguiente (11) es $\varepsilon \lambda \varepsilon \gamma \tau \tau \kappa \sigma \zeta \varsigma^{33}$.

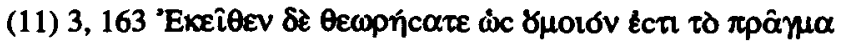

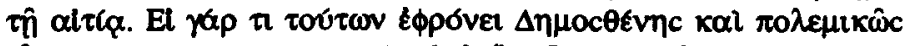

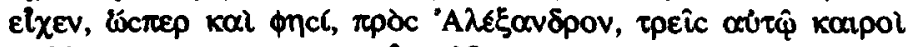

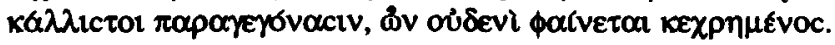

( «Los enemigos aprovechan las ocasiones de atacarse»), «Demóstenes no

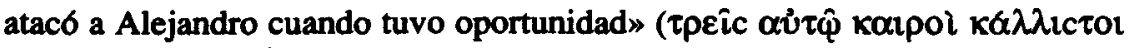

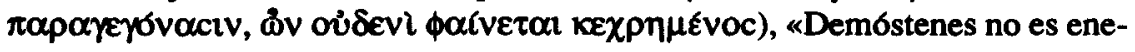

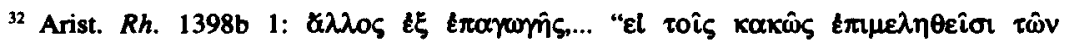

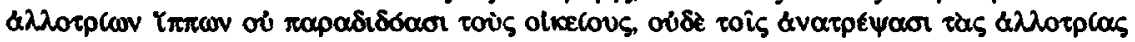

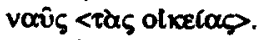

${ }^{33}$ Arist. Rh. 1400 a 15. 


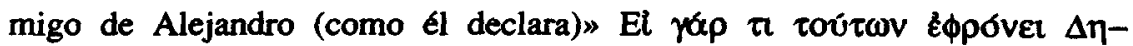

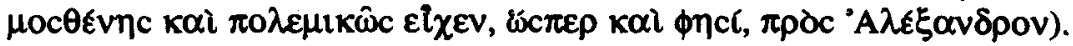

En el tercer gran apartado del discurso Esquines se refiere a la posible réplica de Demóstenes (\$\$168-259). Analiza en primer lugar las cualidades del verdadero demócrata y las del oligarca (\$\$168-176) y demuestra que Demóstenes no es un verdadero demócrata ni por su nacimiento, ni por su conducta, ni por su capacidad de palabra, ni por su valentia. Utiliza en primer lugar una condicional irreal (12), con la que argumenta a partir de contrarios ${ }^{34}$.

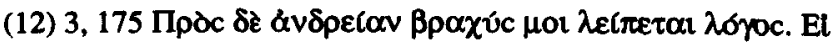

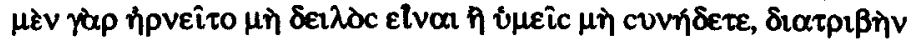

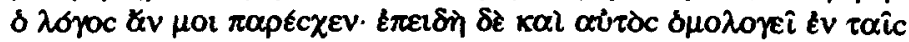

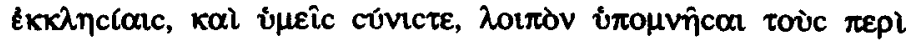

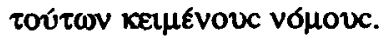

(«Lo evidente no necesita demostración»), «no necesito demostrar la co-

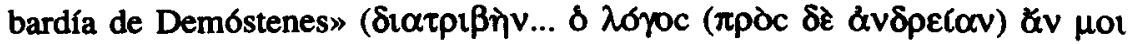

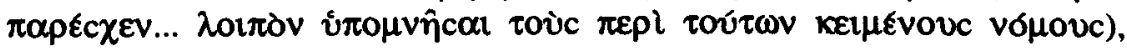

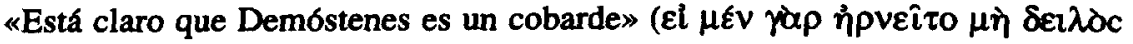

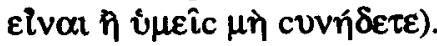

Compara seguidamente ( $\$ 177-198)$ a Demóstenes con los antiguos hombre ilustres que recibían coronas, para evidenciar cómo las recompensas han perdido valor desde que se le dan a gente que no se las merece. En (13) argumenta con un ejemplo en una irreal de pasado.

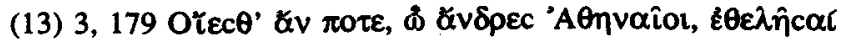

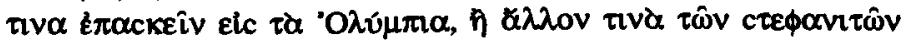

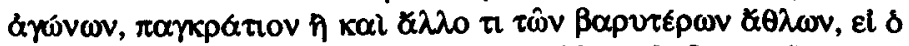

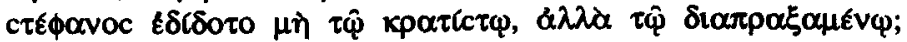

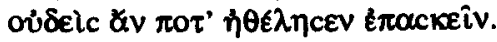

(«Los premios se dan para estimular la virtud»), «se está premiando a los

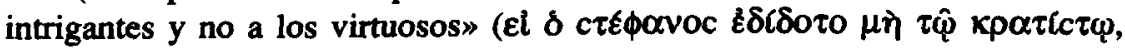

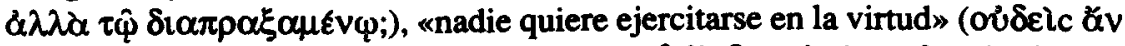

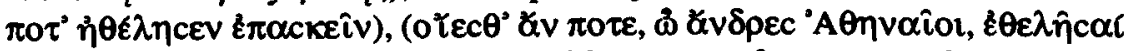

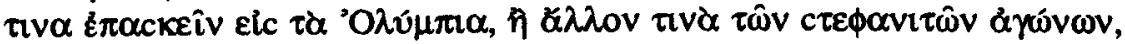

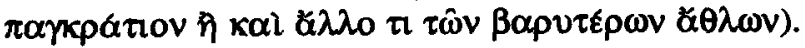




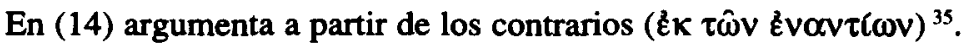

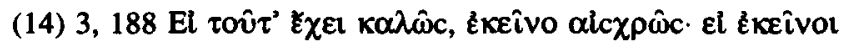

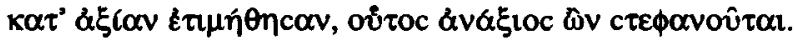

(«honrar a los que son buenos implica no hacerlo con los que no lo son»),

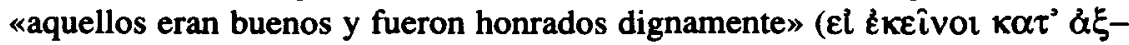

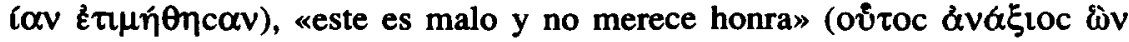

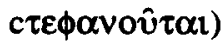

En (15) tenemos un entimema reconstruido sobre una real de presente, en la que la premisa mayor es la oración de relativo. Argumenta basándose en las relaciones recíprocas ${ }^{36}$, si son las leyes las que honran a las personas, las personas deben honrar las leyes.

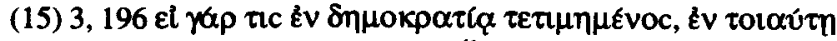

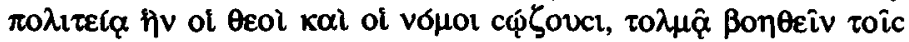

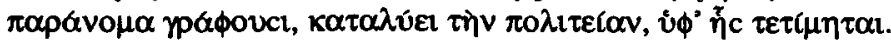

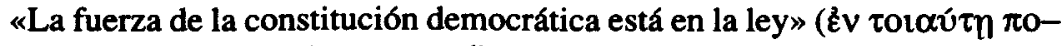

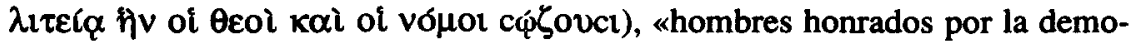

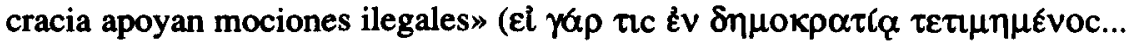

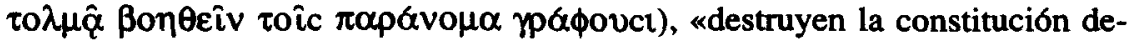

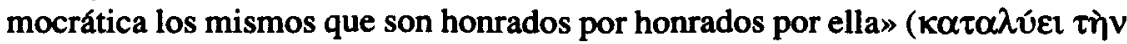
$\pi 0 \lambda \iota \tau \varepsilon i \alpha \nu, \dot{v} \phi^{\prime} \eta \tilde{c} c \tau \varepsilon \tau(\mu \eta \tau \alpha)$.

Se anticipa en \$\$199-229 a la posible réplica de Ctesifonte y advierte a los jueces al respecto. En (16) argumenta con una condicional irreal sobre el ejemplo de un caso igual, semejante o contrario (Arist. $R h$. 1398ba 21) del modo que ya hemos visto.

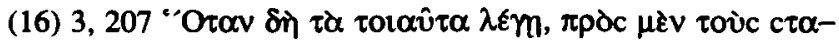

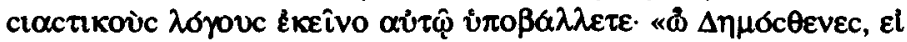

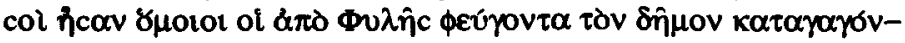

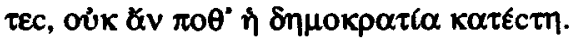

(«Son buenos democratas los que olvidan las afrentas»), «los de File

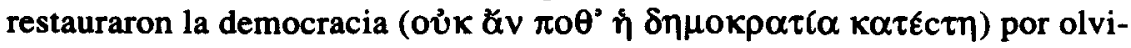

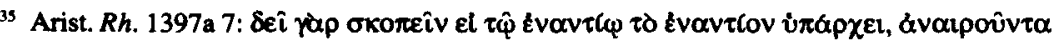
$\mu \mathrm{t} v$ El $\mu$ ì

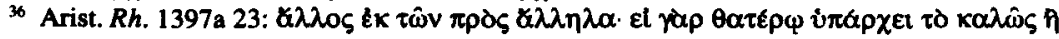

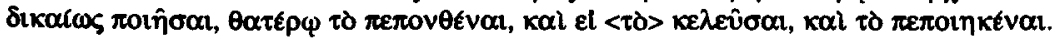


dar las afrentas. Tu no olvidas las afrentas», «los de File son buenos demó-

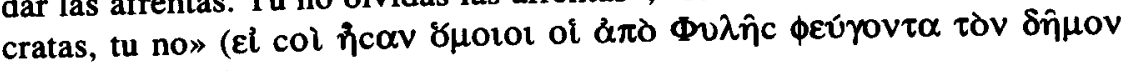

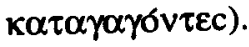

En (17) se defiende de las acusaciones de Demostenes con un argumento refutativo en una irreal de pasado.

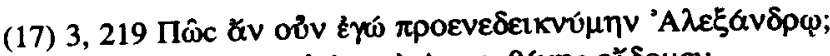

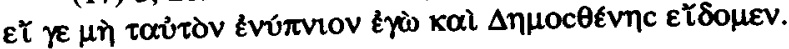

(«No se favorece al que no ha subido al trono»), «yo no vi en sueños la

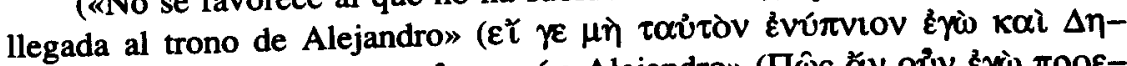

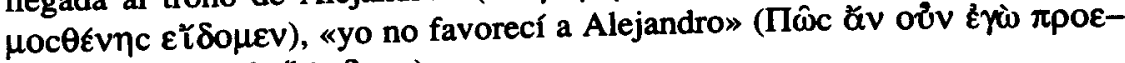

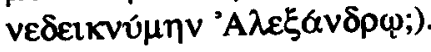

En (18) encontramos una real de pasado en la que tenemos que reconstruir es premisa menor. La premisa mayor se extrae del contexto. Argumenta según se explica en Arist. Rh. 1398a 4 «volviendo contra él lo que dice lo

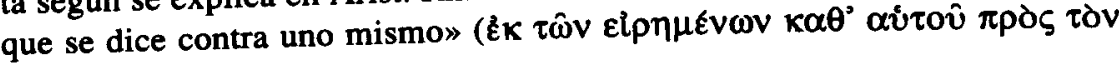
$\varepsilon i \pi \delta \vee \tau \alpha)$.

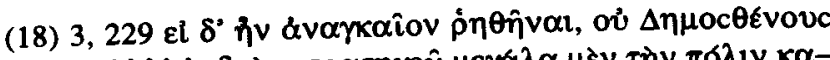

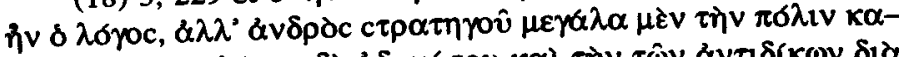

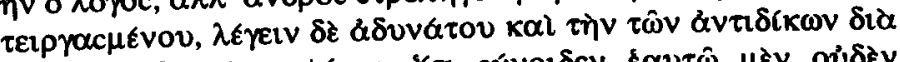

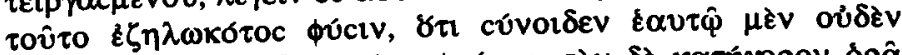

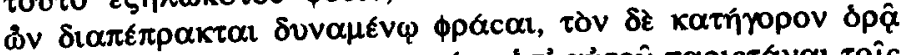

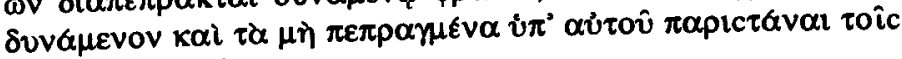

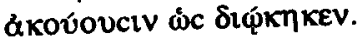

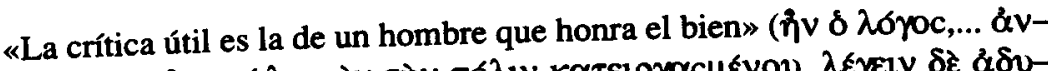

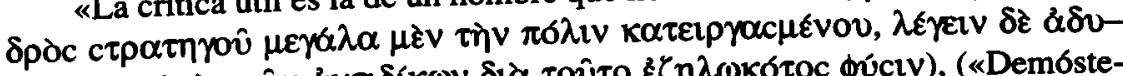

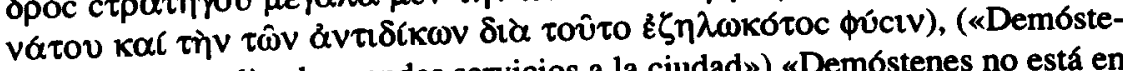
nes no no ha realizado grandes servicios a la ciudad») «Demóstenes no está en condiciones de poder criticar mi facilidad de palabra» (ov̉ $\Delta \eta \mu 0 c \theta \varepsilon v o v c h \vee \delta$ $\lambda \sigma$ бoc).

Esquines termina ( $\$ \$ 230-259)$ haciendo considerar a los jueces lo intolerable que resulta que un hombre como Demóstenes llegue a ser coronado. Encontramos en este último fragmento una condicional irreal de pasado (21) y otras tres condicionales que son expresiones de admiración formuladas como

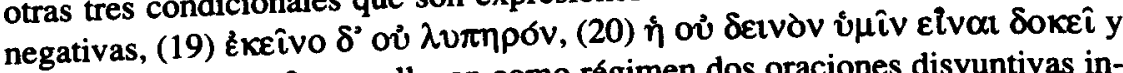

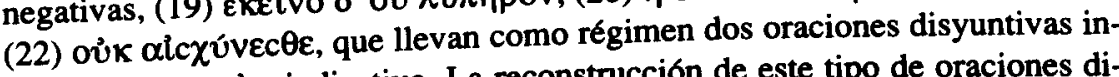
troducidas por $\varepsilon l$ e indicativo. La reconstrucción de este tipo de oraciones difiere del modelo planteado al comienzo, ya que la conclusión del silogismo la 
forman la prótasis y una de las partes de la disyunción, mientras que la otra parte es la premisa menor. En (19) argumenta basándose en las relaciones recíprocas (Arist. Rh. 1397a 23).

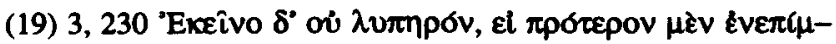

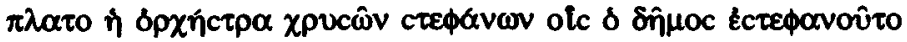

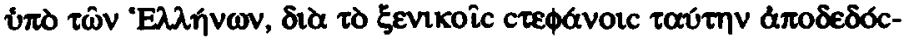

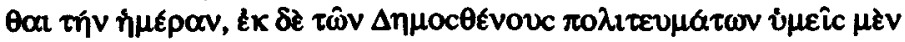

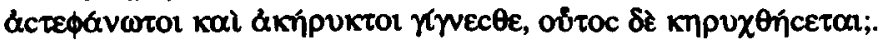

(«Los premios se dan a quien fomenta la virtud»), «Demostenes os ha pri-

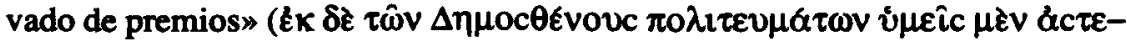

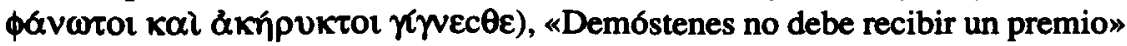

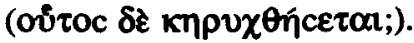

En (20) se fundamenta en la causa (Arist. $R h .1399 \mathrm{~b} 20$ )

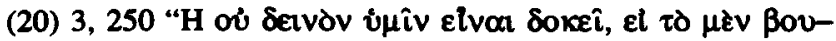

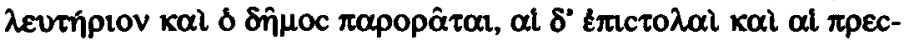

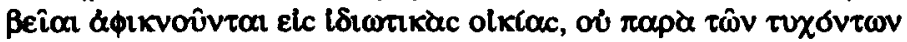

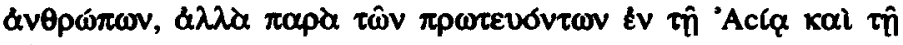

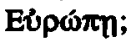

( En una democracia los asuntos que conciernen a todos se resuelven en organismos públicos»), «estos reciben las cartas y las embajadas en casas par-

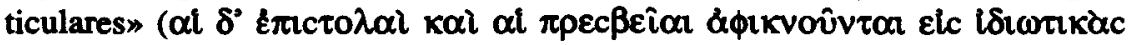

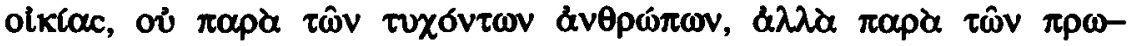

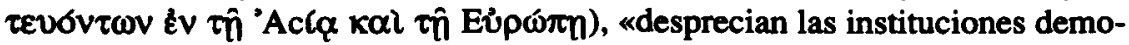

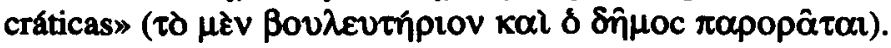

En (21) sigue el mismo tipo de argumentación. \& $\pi \varepsilon \theta 0 \mathrm{~V} \varepsilon \mathrm{V}$

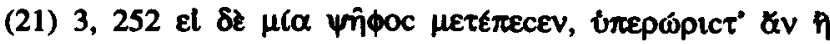

(«Un solo voto puede decidir una sentencia»), «no se decidió la sentencia»

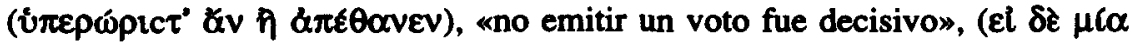

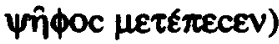

$Y$, por último, en (22) encontramos de nuevo un tratamiento entimemático del ejemplo (Arist. Rh. 1398a 34)

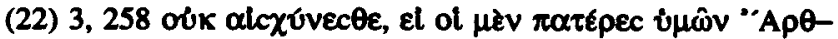

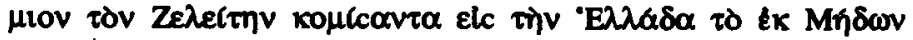




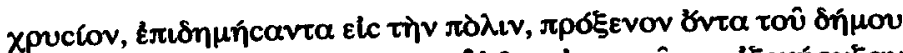

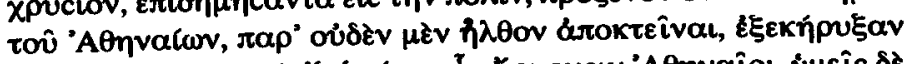

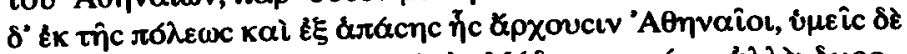

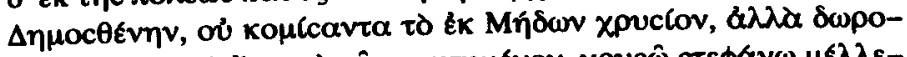

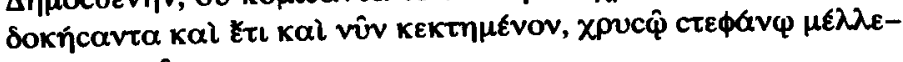

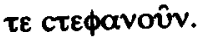

(«Quien acepta oro como soborno no puede recibirlo como premio»), «Atmio de Zeleia, que era huesped del pueblo de Atenas trajo a la Hélade el oro

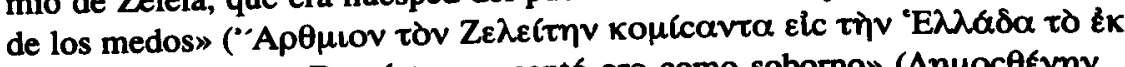

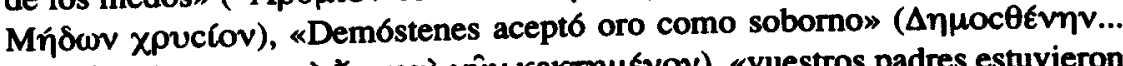

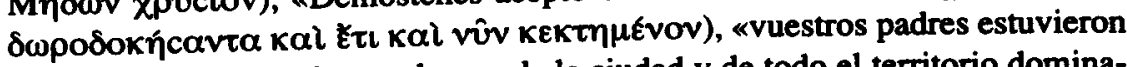
a punto de matarlo y le expulsaron de la ciudad y de todo el territorio domina-

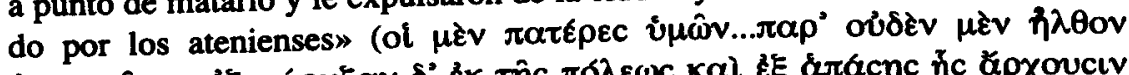

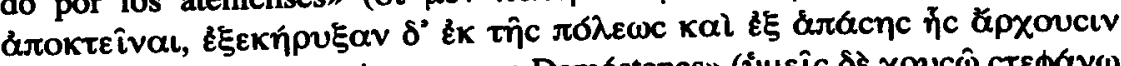

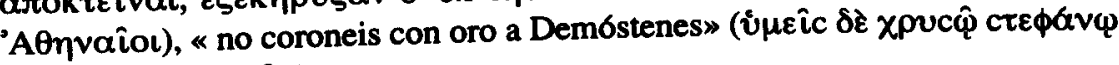

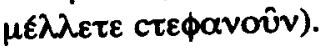

Pasamos ahora al estudio de lo que hemos llamado función, es decir, el ámbito de referencia que domina en los razonamientos lógicos conseguidos mediante entimemas ${ }^{37}$. Este ámbito de referencia ${ }^{38}$ puede residir en el talante

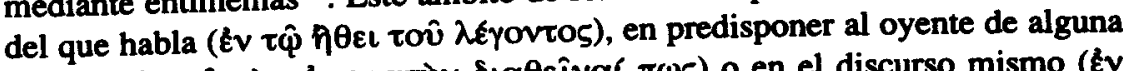

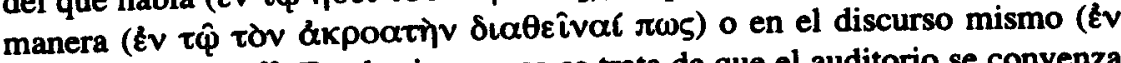
$\alpha u ̛ \tau \hat{\varphi} \tau \hat{\varphi} \lambda \delta \gamma \varphi) »{ }^{39}$. En el primer caso se trata de que el auditorio se convenza de que el hablante tiene credibilidad ( $\alpha \xi\llcorner 0 \pi\llcorner\sigma i \alpha)$, que dice la verdad, y eso

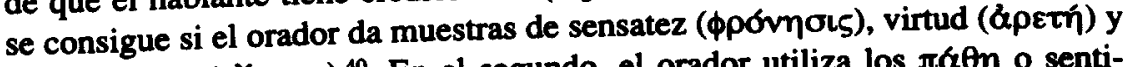
benevolencia ( $\varepsilon$ Úvol $\alpha)^{40}$. En el segundo, el orador utiliza los $\pi \alpha \theta \eta \eta$ o sentimientos humanos como una explicación del comportamiento y también para provocar determinados sentimientos en el auditorio «porque las pasiones son, ciertamente, las causantes de que los hombres se hagan volubles y cam-

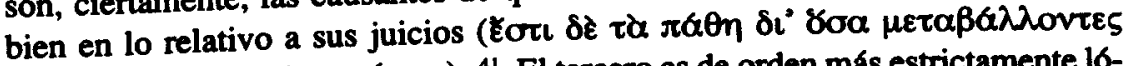

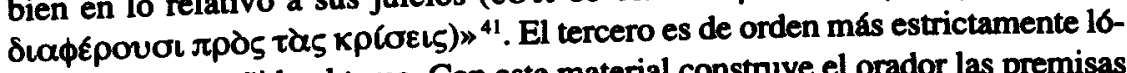
gico, es decir, ceñido al tema. Con este material construye el orador las premisas

37 Ver al respecto HeLLWIG, A., (1973), Untersuchungen zur Theorie der Rhetorik bei Platon und Aristoteles, Grttingen, p. 234 y ss.

38 Arist. $R h .1355 b 35$.

39 Arist. Rh. 1356a13.

to Arist. Rh. 1378a 6-8.

41 Arist. Rh. 1378a 20. Cf. al respecto ForTenwauch, W. (1992), «Aristotle on Persuasion through Characterw, Rhetorica 10 , pp. 207-224. 
de naturaleza probable ${ }^{42}$ o elkó $\tau \alpha$ sobre las que argumenta y con las que supone que el auditorio está de acuerdo ${ }^{43}$. Nos interesa ahora ver cómo se refleja esto en los entimemas reconstruidos a partir de condicionales en el Contra Ctesifonte y ponerlo en relación con el momento del discurso, con el tipo de condicionales y con los tópicos.

Hemos visto que, en la primera parte ( $\$ 9-48)$, al mostrar la disconformidad del decreto con las leyes vigentes argumenta Esquines con condicionales

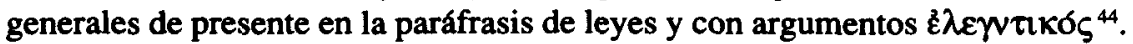
En todas ellas la argumentación se apoya en la leyes (1) y (2) y en la confianza del orador en los mecanismos democráticos ((4)»Las constituciones no admiten leyes contradictorias», (5) «Las ciudades evitan las leyes contradictorias suprimiendo una de ellas»). Con ello intenta suscitar su propia credibilidad, dando muestras de benevolencia ${ }^{45}$, una de cuyas manifestaciones es la tolerancia y el respeto hacia el auditorio, que se traduce en lealtad a la constitución ${ }^{46}$.

Cuando pasa ya a la acusación concreta de la actuación política de Demóstenes (\$\$49-167), las premisas sobre las que argumenta ((6) «Firmar la paz, no solos, sino con la conformidad de los otros estados es lo que produce una situación de hegemonía», (7) «Las situaciones de extremo peligro exigen un comportamiento extraordinario», (8) «El recurso a la huida es propio de situaciones de extremo peligro», (9) «Son enemigos de la ciudad los que permiten que corra riesgos innecesarios», (10) «No debe seguir dirigiendo quien ha llevado al fracaso aquello que dirigía» y (11) «Los enemigos aprovechan las ocasiones de atacarse») se refieren a lo que acontece la mayoría de las veces, es decir, se ciñen al asunto mismo.

En el anticipo a la posible réplica de Demóstenes (\$\$168-198), Esquines intenta hacer reflexionar a los jueces sobre las consecuencias de su conducta y apela a una de las pasiones que Aristóteles considera «honrosa y propia de hombres honrados» ${ }^{47}$, la emulación ((12) «Lo evidente no necesita demostración», (13) «Los premios se dan para estimular la virtud», (14) «honrar a los que son buenos implica no hacerlo con los que no lo son» y (15) «La fuerza de la constitución democrática está en la ley»)). Pretende evitar que con su comportamiento retraigan a alguien de hacer el bien.

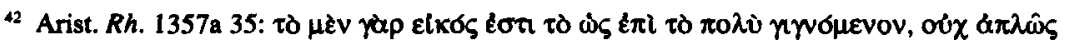

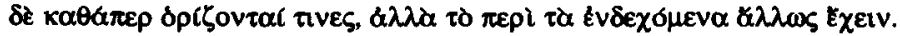

${ }^{43}$ Cf. Grimaldi (1980), p. 104.

44 Arist. Rh. 1400a 15.

45 Arist. Rh. 1356a 1-13

46 Cf. Racionero (1990), p. 176.

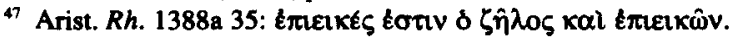


En el pasaje siguiente, en el que se anticipa Esquines al discurso que pronunciará Ctesifonte (\$\$199-229) vuelve a dominar la función de provocar la credibilidad del orador, dando muestras de sensatez ((16) «Son buenos demócratas los que olvidan las afrentas», (17) «No se favorece al que no ha subido al trono», (18) «La crítica útil es la de un hombre que obra el bien»).

Al final del discurso ( $\$ \$ 230-259)$ encontramos tres condicionales dependientes de expresiones de admiración formuladas como negativas, que vuelven otra vez al ámbito de provocar sentimientos en los jueces. En primer lugar a no dejarse llevar por el temor ${ }^{48}$ en su decisión ((21) «Un solo voto puede decidir una sentencia»), con las consecuencias que de eso se pueden derivar ((20) «En una democracia los asuntos que conciernen a todos se resuelven en organismos públicos») y a que, con su actuación, debiliten en los jovenes el afán de emulación ((19) «Los premios se dan a quien fomenta la virtud», (22) «Quien acepta oro como soborno no puede recibirlo como premio»).

En conclusión podemos afirmar que, tanto en la forma como en la función, predomina en cada parte del discurso un tipo de argumento. Así, en el momento en que Esquines quiere justificar su decisión de haber presentado una acusación de ilegalidad al decreto de Ctesifonte, busca apoyo para su argu-

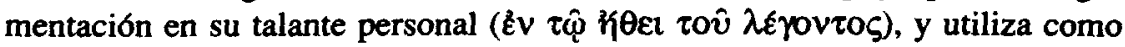
tipo de argumentos la paráfrasis de leyes, (1) y (2), y los refutativos (4) y (5). Cuando acusa directamente a Demóstenes de haber llevado a la ciudad a situaciones de extremo peligro, predomina la función referida al asunto mismo ( $\varepsilon^{2} \mathrm{v}$

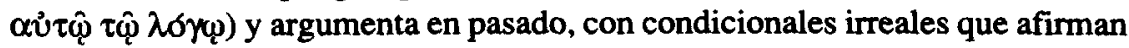
en el plano de lo real lo que es una causa solo posible, (6), (7) y (8), y con reales, (9) y (10). Esto confirma la afirmación de Aristóteles de que el tiempo adecuado para la acción judicial es el pasado, «ya que siempre se hacen acusaciones o defensas en relación con acontecimientos ya sucedidos» ${ }^{49}$. Y en la última parte, cuando procura influir en la decisión de los jueces, busca premi-

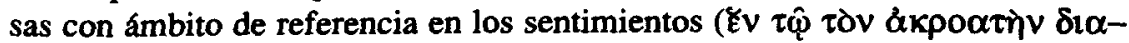
$\theta \varepsilon i v \alpha(\pi \omega \zeta)$ y utiliza para ello condicionales disyuntivas, dependientes de expresiones de admiración (19), (20), (22).

Un análisis del discurso, como el que acabamos de ofrecer, puede servir de ayuda tanto para profundizar en el estudio de los mecanismos de persuasión de los oradores, como para demostrar el carácter eminentemente práctico de la obra aristotélica y contribuir así a una nueva la lectura de la Retórica.

48 Arist. Rh. 1382a 20.

49 Arist. Rh. $1358 \mathrm{~b} 16$. 